\title{
LA EVALUACIÓN FORMATIVA EN EL AULA UNIVERSITARIA
}

The formative assessment in the university classroom

\section{A avaliação formativa na sala de aula universitária}

\section{Guillermo Alejandro Campos Cancino}

(1) Universidad de Valladolid, España. Teléfono: +34 644487231. Correo electrónico: guillermoalejandro.campos@uva.es

\section{Resumen}

En esta investigación, se expone una experiencia práctica con alumnado universitario, donde se sistematizó en dos sesiones de clase el análisis de una evaluación formativa. En este sentido, la mirada se centra en las interacciones que se producen en el interior del aula y se destaca que la evaluación debe trascender al proceso de control y asumir el aspecto formativo, centrado en el aprendizaje de los estudiantes y en la mejora.

El estudio que se realizó aporta información descriptiva e interpretativa sobre una práctica que persigue visualizar y destacar la importancia del proceso, propiciar el diálogo, la participación y las retroalimentaciones entre pares y entre profesor - alumno dentro del aula. De este modo, se favorece un aprendizaje eficaz y significativo.

Palabras clave: Evaluación formativa; estudiantes universitarios; educación

\begin{abstract}
In this research, a practical experience with university students is exposed, where the analysis of a formative assement was systematized in two sessions of class. In this sense, the focus is on the interactions that take place inside the classroom and it is emphasized that the evaluation must go beyond the control process and assume the formative aspect, centered on student learning and improvement.

The study that was conducted provides descriptive and interpretative information about a practice that seeks to visualize and highlight the importance of the process, promote dialogue, participation and feedback between peers and between teacher - student in the classroom. In this way, effective and meaningful learning is favored.

Keywords: Formative assement; university students; education
\end{abstract}




\section{Resumo}

Nesta pesquisa, expõe-se uma experiência prática com estudantes universitários, onde foi sistematizada em duas aulas a análise de uma avaliação formativa . Neste sentido, o foco está nas interações que ocorrem dentro da sala de aula e enfatiza-se que a avaliação deve ir além do processo de controlo e assumir o aspecto formativo, centrado na aprendizagem dos alunos e na sua melhoria.

O estudo realizado fornece informação descritiva e interpretativa sobre uma prática que procura ver e destacar a importância do processo, promover o diálogo, a participação e os feedbacks entre pares e entre professor - aluno dentro da sala de aula. Desta forma, favorece-se uma aprendizagem eficaz e significativa.

Palavras-chave: Avaliação formativa; estudantes universitários; educação

\section{Introducción}

Para comenzar, y a modo de preámbulo, es importante destacar que la evaluación educativa tradicional era entendida o reducida a un acto final. No obstante, hoy en día ese paradigma no logra abarcar la totalidad del concepto evaluar (Dochy, Segers, \& Sluijsmans, 1999). Por lo tanto, se debe subrayar que desde el inicio hasta el cierre de la clase y en la medida en que los estudiantes van aprendiendo, el docente realiza reiterados procesos valorativos del nivel de comprensión y aprendizaje de sus alumnos. La evaluación es un término polisémico y que ha evolucionado con el tiempo (Córdoba, 2006). Evaluar fue asociado a un momento particular y donde el profesor realizaba la acción de calificar mediante una nota. En la actualidad, la evaluación se considera un proceso cíclico y que condiciona y es condicionado por la enseñanza y el aprendizaje. Por consiguiente, es una tarea más compleja y más amplia que solo calificar.

López (2013) hace referencia a la evaluación como un ejercicio que debe servir para mejorar el proceso de enseñanza-aprendizaje. En este sentido, la evaluación formativa tiene esa finalidad, además de permitir tomar distancia de las prácticas evaluativas más tradicionales. A continuación, se presenta una experiencia educativa en un aula universitaria donde se expone el desarrollo de una evaluación formativa.

\section{Contextualización}

La experiencia de evaluación se realizó en el Grado de Educación Primaria, en la asignatura de Métodos de Investigación e Innovación en Educación. Es un curso mixto 
y constituido por 46 estudiantes. Esta asignatura se imparte en segundo año de la carrera, durante el segundo cuatrimestre y tiene una carga lectiva de 6 créditos.

El clima dentro del salón de clase es bueno y los estudiantes realizan las actividades propuestas y tienen una actitud participativa. Los contenidos se planifican clase a clase y se extraen del libro: Metodología de la Investigación de Hernández, FernándezCollado, \& Baptista (2014), el cual facilita un cronograma de los aprendizajes que hay que abarcar. Además, se debe señalar que las aulas disponen de recursos tecnológicos que permiten la proyección del contenido como material audiovisual, también los estudiantes disponen de red "wifi" y los contenidos de clase y el material complementario se suben al campus virtual propio de la universidad.

\section{Diseño}

Una sesión de clase tiene una duración de una hora cronológica, por lo tanto, el control del tiempo es importante. La clase se divide en tres momentos principales: Inicio, desarrollo y cierre. Estos están determinados por una asignación de tiempo y se sistematizan en fases que se inician con una serie de interrogantes de respuesta abierta que incentivan la participación, el diálogo y una evaluación formativa sobre los conocimientos previos de los estudiantes, sus opiniones, los conceptos importantes que han aprendido en esa sesión, la aplicación de los aspectos teóricos y una valoración de las técnicas de enseñanza del profesor. Todo esto se detalla en el punto de desarrollo.

A continuación, en la tabla 1 se resume el diseño:

Tabla 1.

Resumen diseño

\begin{tabular}{ll}
\hline Categorías & Descripción \\
\hline 1.- Técnica & Observación del estudiante. \\
2.- Instrumento & Preguntas de respuesta abierta (hechas de forma oral). \\
\hline
\end{tabular}

Nota: Elaboración propia.

\section{Desarrollo}

En las sesiones, se realizó una evaluación formativa concretizada en dos aspectos: a) En preguntas directas y abiertas al estudiante (de forma oral), las que deberán ser respondidas y reflejarán un diagnóstico sobre los conocimientos previos del alumno, opiniones o valoraciones de aspectos positivos o negativos de las técnicas de enseñanza desarrolladas en las sesiones de clase. $\mathrm{Y}$ b) en la verificación del grado de entendimiento de lo expuesto y si se relaciona con lo que ya sabía el alumno, es decir si 
los aprendizajes están siendo significativos. A continuación, se detalla la estructura de la clase:

1-. Inicio (15 minutos): -Presentación del objetivo. Ej.: Presentar los tipos de investigación cuantitativa. -Motivación. Ej.: ¿Por qué es útil este contenido? Como profesores ¿podrán usarlo en el futuro?

2-. Desarrollo (35 minutos): -Activación de conocimientos previos. Ej.: ¿Qué saben sobre este contenido? ¿Conocen algún ejemplo de investigación cuantitativa? Contenido. Ej. Se expone el contenido y si es necesario se complementa con una guía de aprendizaje. -Actividad práctica. Ej.: Se presenta un caso donde se debe establecer a qué tipo de investigación cuantitativa corresponde. Tiene un tiempo de 5 minutos y siempre se corrige con la participación de voluntarios o asignación al azar.

3-. Cierre (10 minutos): -Sistematización. Ej. ¿Qué les pareció cómo se desarrolló la clase? ¿Qué aspectos les gustaría destacar o cuáles mejorar? -Teorización. Ej. ¿Qué conceptos importantes se revisaron hoy? ¿Defina algún concepto que aprendió hoy?

\section{Evaluación}

La fase de evaluación se llevó a cabo mediante la reflexión del docente sobre su quehacer en el aula, se consideraron las ventajas e inconvenientes del desarrolla de la experiencia como se visualiza en la Figura 1.

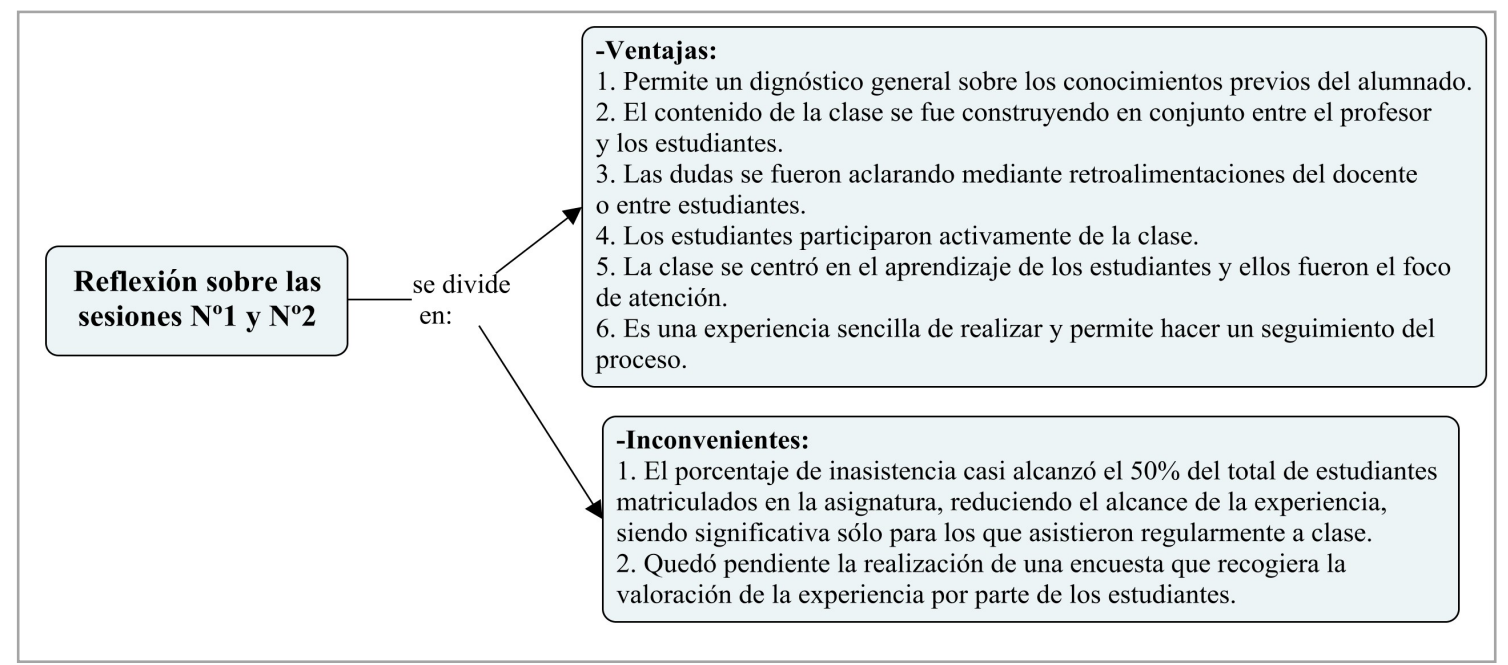

Figura 1. Evaluación de la experiencia 


\section{Conclusiones}

El desarrollo de la experiencia educativa se centró en la evaluación formativa, la cual permitió una comprensión más amplia del aprendizaje de los estudiantes en los siguientes aspectos:

a) Hubo un seguimiento del proceso de enseñanza-aprendizaje a través de la realización de las distintas preguntas abiertas, las cuales invitaban a la participación de los estudiantes en el desarrollo de la clase.

b) La evaluación formativa permitió flexibilidad, porque se adaptó a las necesidades del grupo curso. En el caso de estas sesiones, se llevó a cabo mediante la retroalimentación y el diálogo establecido dentro de la clase entre profesor-alumno y entre pares.

Y finalmente c) la experiencia permitió destacar la importancia del proceso y tratar cualquier concepto equivocado o duda que los estudiantes de manera individual o colectiva hayan tenido. Además, facilitó la verificación de la comprensión del alumnado.

En el futuro sería interesante implantar esta experiencia en otros niveles educativos o grados universitarios. A modo de cierre, me gustaría dejar planteada la siguiente pregunta: ¿se obtendrían resultados igual de positivos?

\section{Referencias}

Córdoba, F. (2006). La evaluación de los estudiantes: una discusión abierta. Revista Iberoamericana de Educación, 39(7), 1-9.

Dochy, F., Segers, M., \& Sluijsmans, D. (1999). The Use of Self-, Peer and Coassessment in Higher Education: a review. Studies in HigherEducation, 24(3), $331-350$.

López, V. (2013). Nuevas Perspectivas sobre Evaluación en Educación Física. Revista de Educación Física, 29(3), 1-10.

Hernández, R., Fernández-Collado, C., \& Baptista, P. (2014). Metodología de la investigación. México: McGraw-Hill Interamericana. 